\title{
Instantaneous Sonophotocatalytic Degradation of
}

\section{Tetracycline over NU-1000@ZnIn $\mathrm{S}_{4}$ Core-Shell Nanorods as a \\ Robust and Eco-Friendly Catalyst}

Reza Abazari, ${ }^{\dagger}$ Soheila Sanati, ${ }^{\dagger}$ Ali Morsali, ${ }^{*}{ }^{\dagger}$ and Alexander M. Kirillov ${ }^{\ddagger}, \S$

${ }^{\dagger}$ Department of Chemistry, Faculty of Basic Sciences, Tarbiat Modares University, Tehran, 14115-175, Iran

‡Centro de Química Estrutural, Instituto Superior Técnico, Universidade de Lisboa, Av. Rovisco Pais, 1049-001 Lisbon, Portugal

${ }^{\S}$ Research Institute of Chemistry, Peoples' Friendship University of Russia (RUDN University), 6 Miklukho-Maklaya st., Moscow, 117198, Russia 


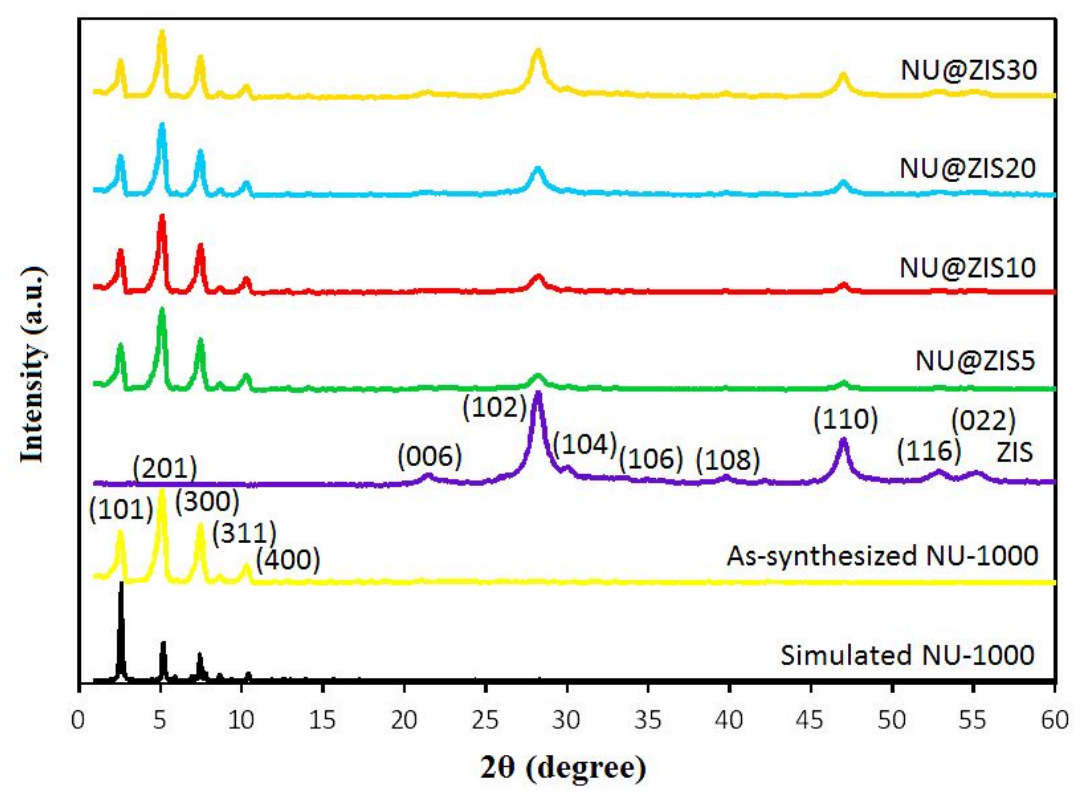

Figure S1. PXRD patterns NU-1000 (simulated from single-crystal X-ray data and assynthesized), ZIS, and NU@ZIS nanocomposites.

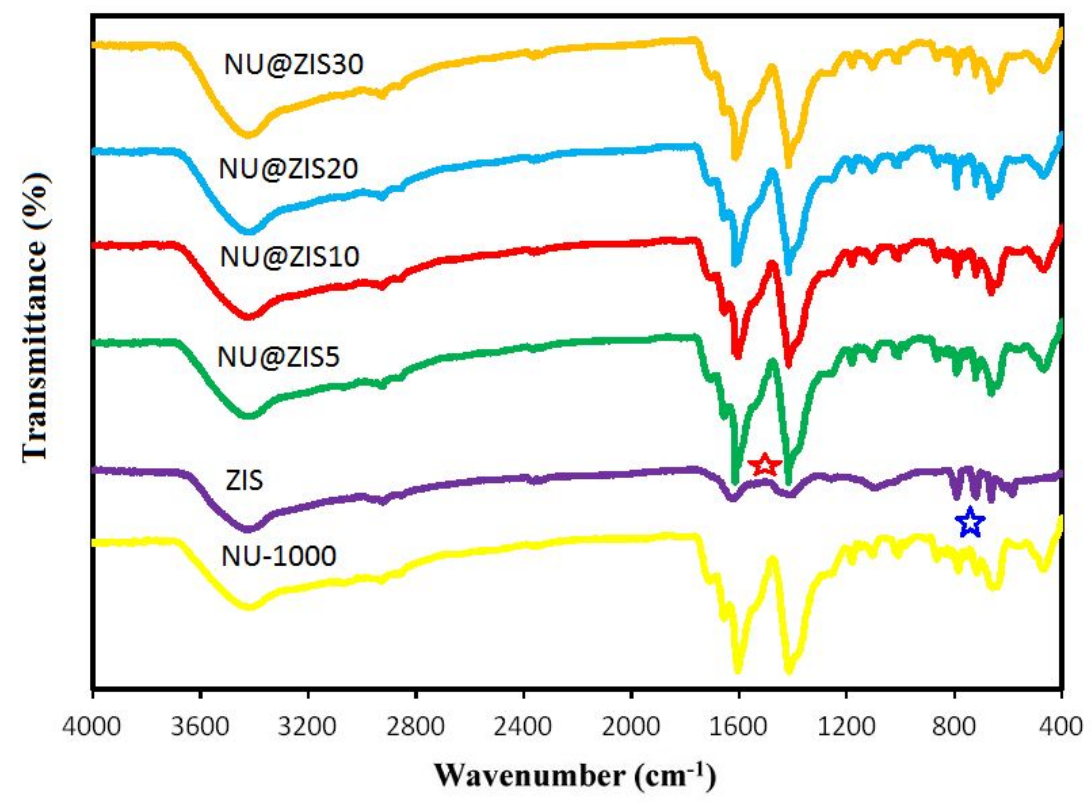

Figure S2. FT-IR spectra of NU-1000, ZIS, and NU@ZIS nanocomposites. 


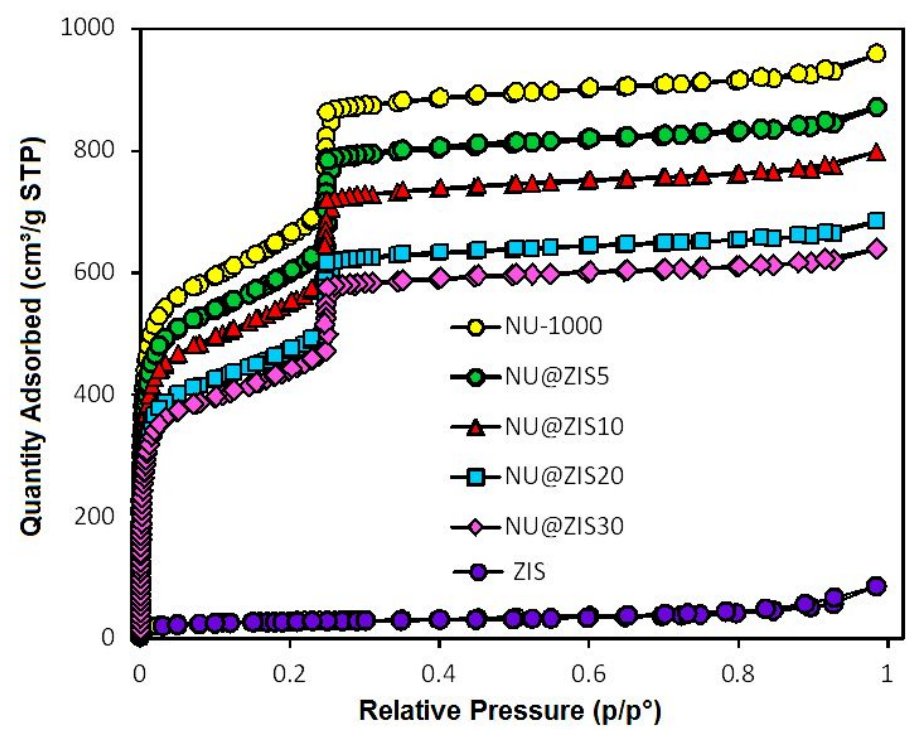

Figure S3. Isotherms of nitrogen adsorption-desorption at $77 \mathrm{~K}$ for the as-synthesized NU-1000, ZIS, and NU@ZIS nanocomposites.

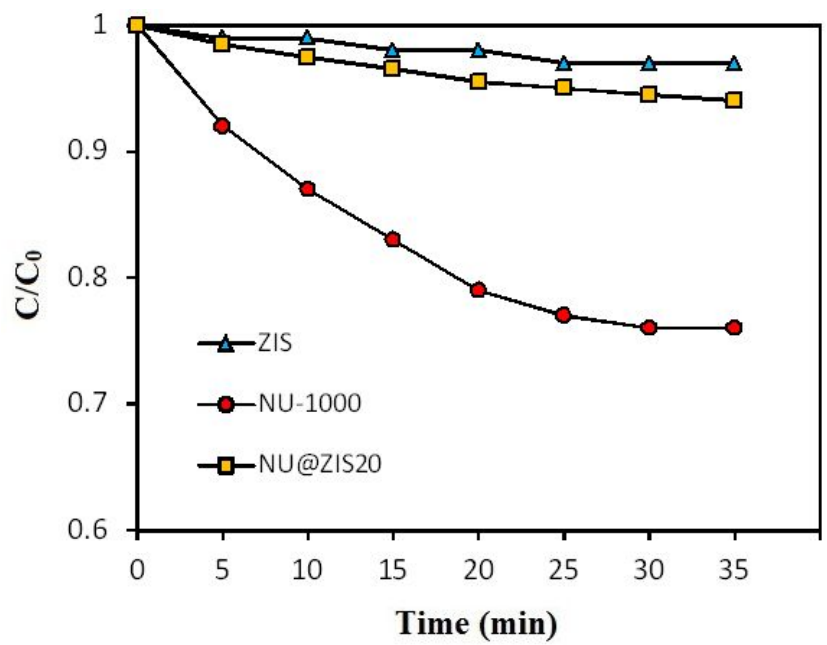

Figure S4. Removal efficiency of TC by NU-1000, ZIS, and NU@ZIS20 nanocomposites in the absence of light and ultrasound waves after $35 \mathrm{~min}$ (catalyst loading $=0.2 \mathrm{~g} \cdot \mathrm{L}^{-1}, \mathrm{C}_{0}(\mathrm{TC})=20$

$$
\left.\mathrm{mg} \cdot \mathrm{L}^{-1}, \mathrm{pH}=7\right) \text {. }
$$




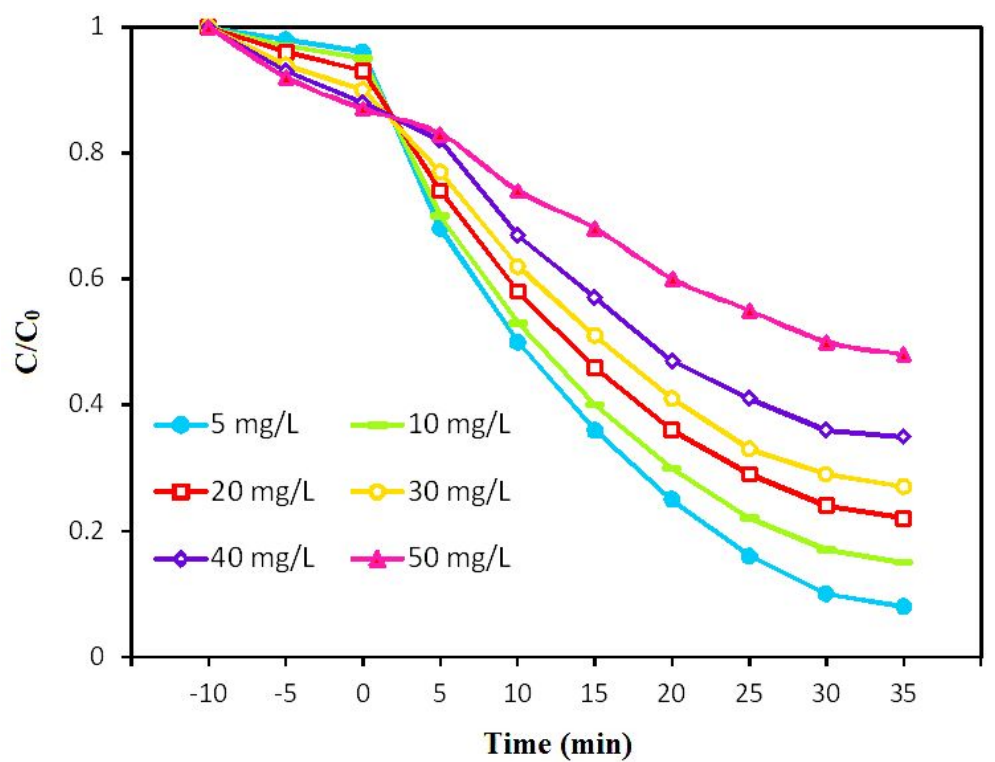

Figure S5. Effect of tetracycline initial concentration, $\mathrm{C}_{0}(\mathrm{TC})$, on the sonophotocatalytic degradation over NU@ZIS20 nanocomposite under a 300 W halogen lamp irradiation (catalyst loading $\left.=0.2 \mathrm{~g} \cdot \mathrm{L}^{-1}, \mathrm{pH}=7\right)$

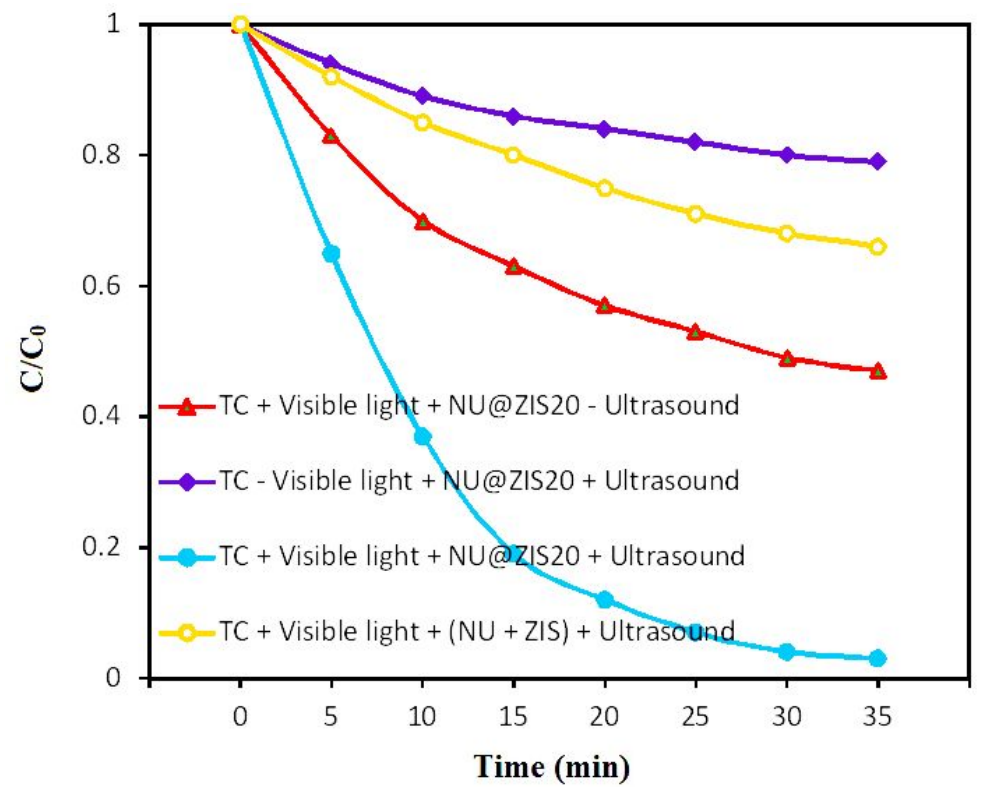

Figure S6. Degradation of tetracycline over NU@ZIS20 nanocomposite and physical mixture of $\mathrm{NU}-1000+$ ZIS under different reaction conditions (catalyst loading $=0.2 \mathrm{~g} \cdot \mathrm{L}^{-1}, \mathrm{C}_{0}(\mathrm{TC})=50 \mathrm{mg} \cdot \mathrm{L}^{-1}$, $\mathrm{pH}=7)$. 


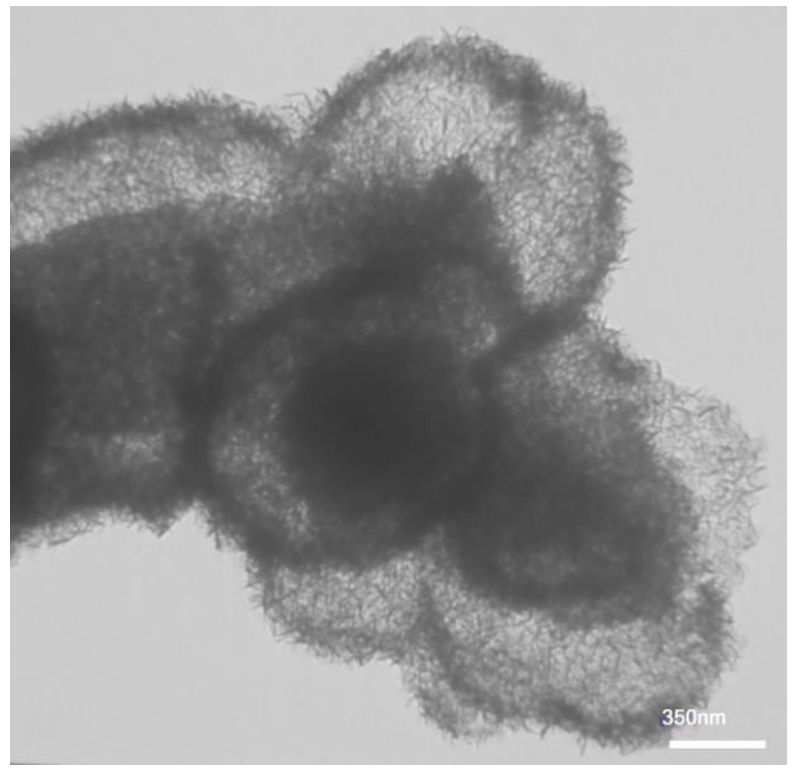

Figure S7. TEM image of NU@ZIS20 nanocomposite after degradation of TC under ultrasonic waves with irradiation frequency of $60 \mathrm{kHz}$.

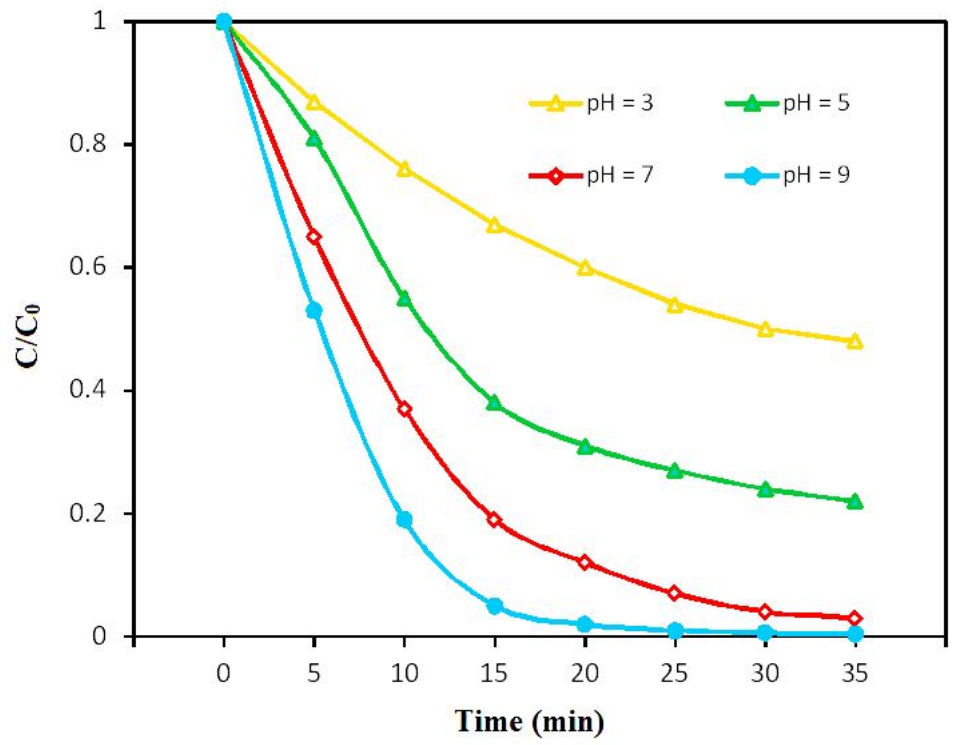

Figure S8. Solution pH effect on sonophotocatalytic tetracycline degradation over NU@ZIS20 nanocomposite under a $300 \mathrm{~W}$ halogen lamp irradiation (catalyst loading $=0.2 \mathrm{~g} \cdot \mathrm{L}^{-1}, \mathrm{C}_{0}(\mathrm{TC})=50$ $\left.\mathrm{mg} \cdot \mathrm{L}^{-1}\right)$ 


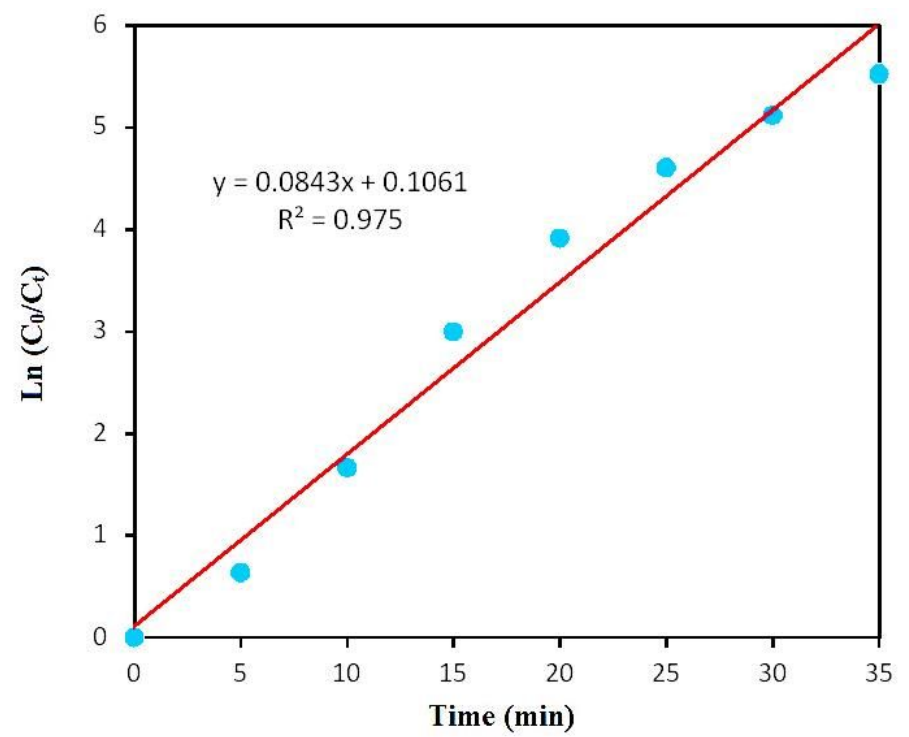

Figure S9. Reaction kinetics (pseudo-first order) for sonophotocatalytic tetracycline degradation over NU@ZIS20 nanocomposite under a 300 W halogen lamp irradiation (catalyst loading = 0.2

$$
\mathrm{g} \cdot \mathrm{L}^{-1}, \mathrm{C}_{0}(\mathrm{TC})=50 \mathrm{mg} \cdot \mathrm{L}^{-1}, \mathrm{pH}=9 \text { ). }
$$
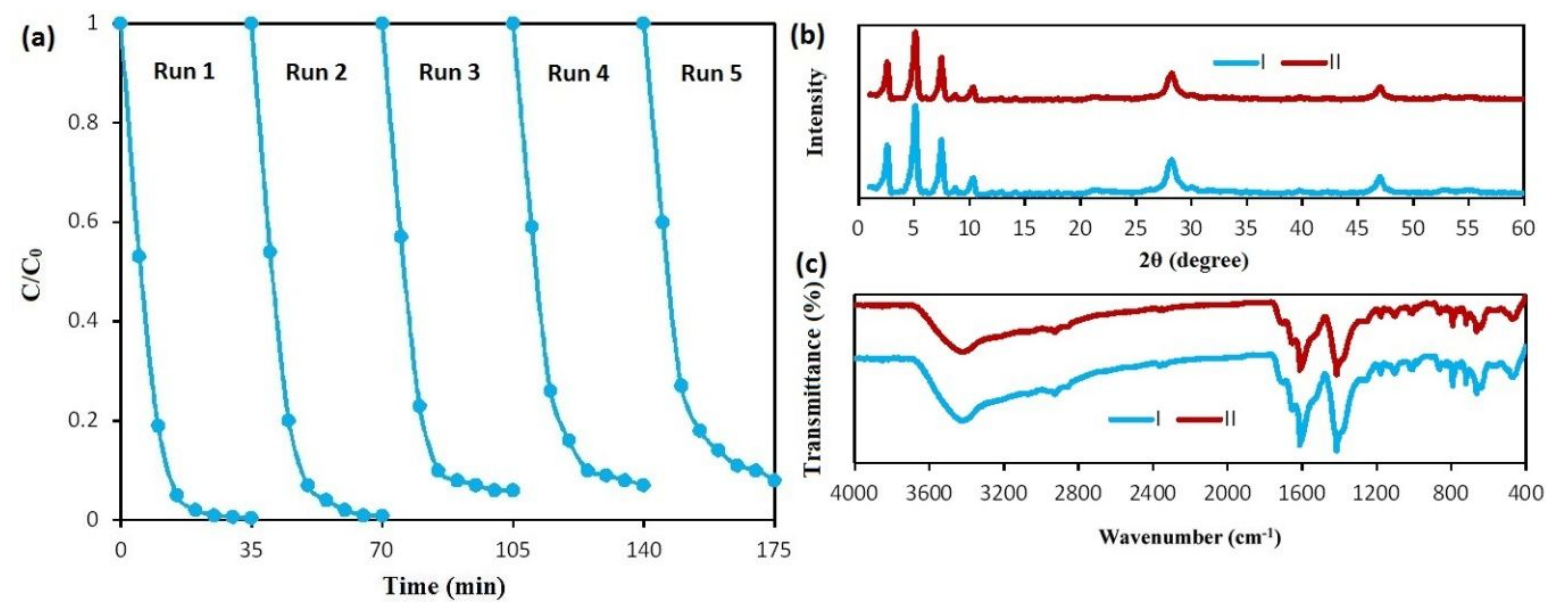

Figure S10. (a) Recycling of NU@ZIS20 nanocomposite catalyst in sonophotocatalytic tetracycline degradation under a $300 \mathrm{~W}$ halogen lamp irradiation (catalyst loading $=0.2 \mathrm{~g} \cdot \mathrm{L}^{-1}$, $\mathrm{C}_{0}(\mathrm{TC})=50 \mathrm{mg} \cdot \mathrm{L}^{-1}, \mathrm{pH}=9$ ). (b) PXRD patterns and (c) FT-IR spectra of NU@ZIS20 before (I) and after (II) sonophotocatalytic tests. 


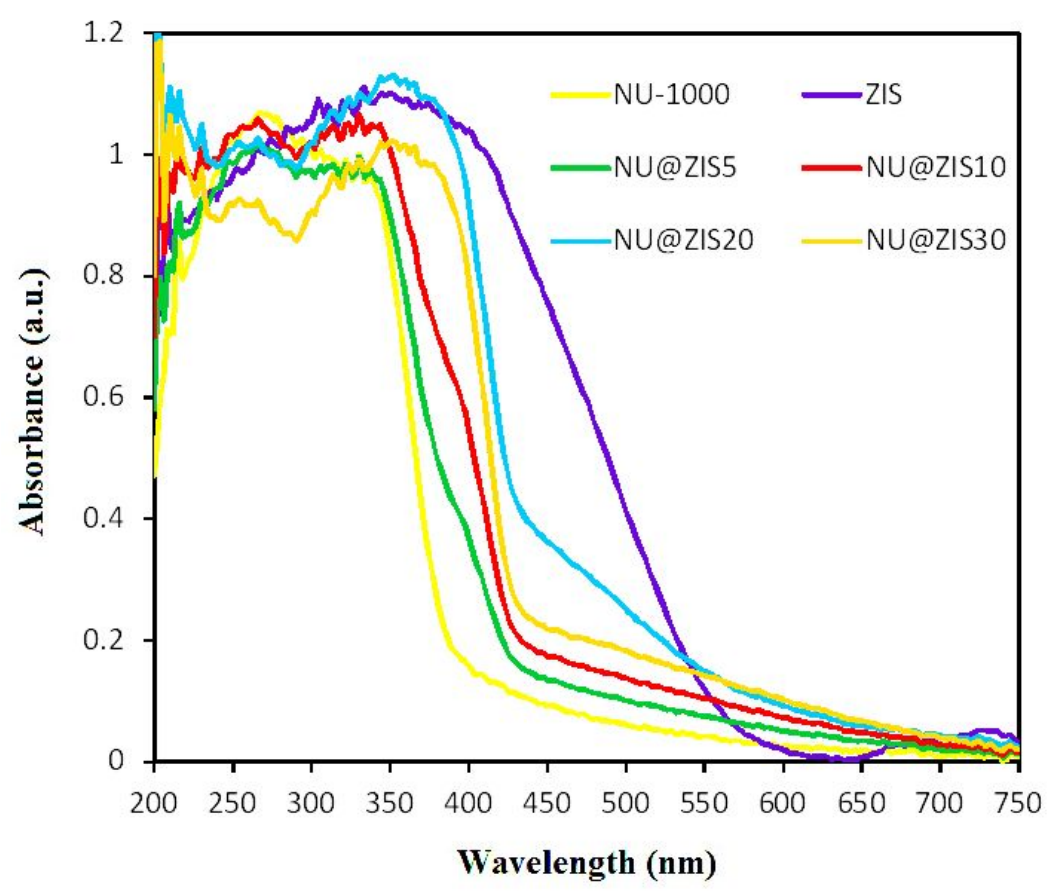

Figure S11. UV-Vis diffuse reflectance spectra of NU-1000, ZIS, and NU@ZISX nanocomposites

$$
(X=5-30 w t \%) .
$$
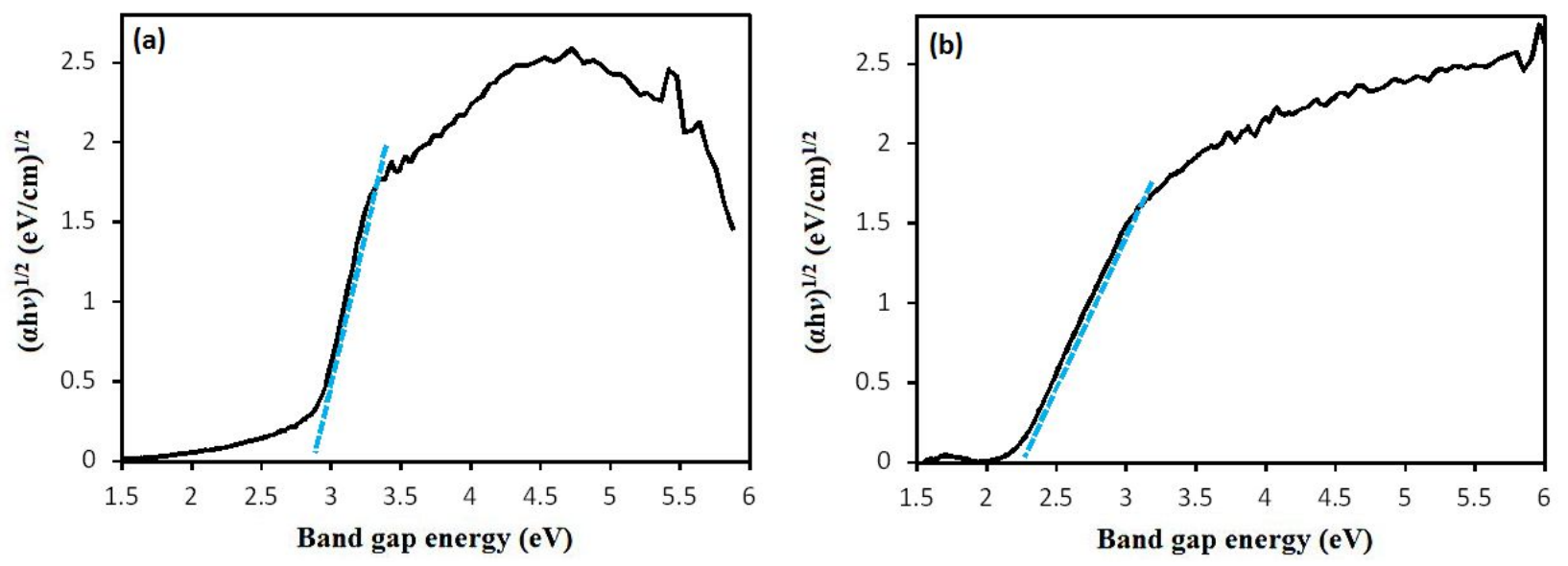

Figure S12. Band edges based on Tauc relationship: (a) NU-1000 and (b) ZIS. 


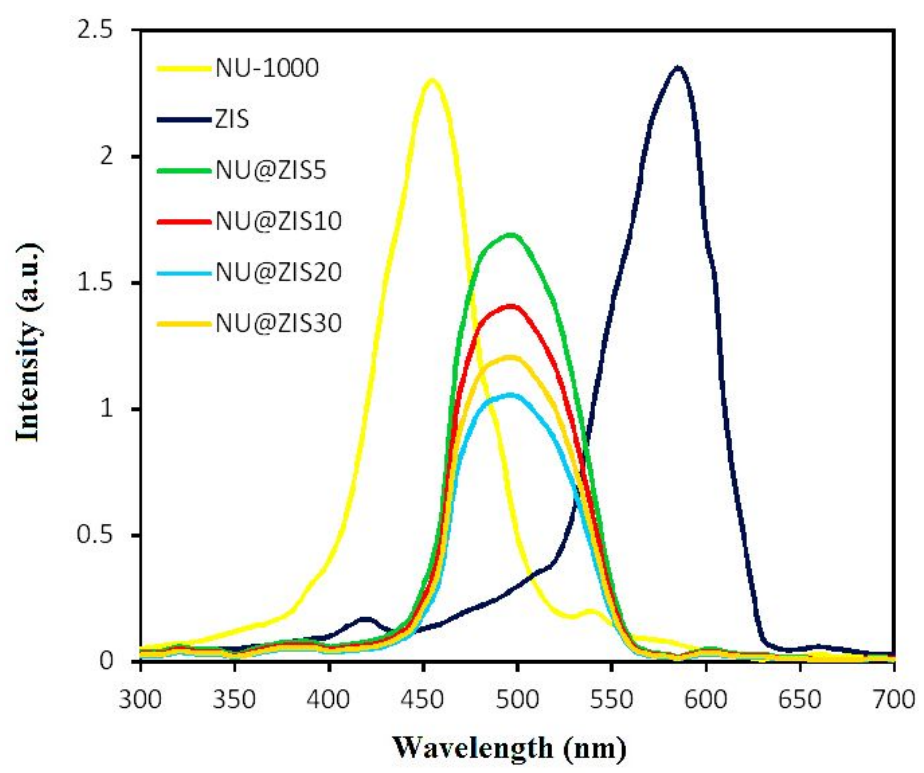

Figure S13. Photoluminescence spectra of NU-1000, ZIS, and NU@ZISX nanocomposites (X=5, 10,20 and $\left.30 \mathrm{wt} \% ; \lambda_{\text {ex. }}=345 \mathrm{~nm}\right)$.
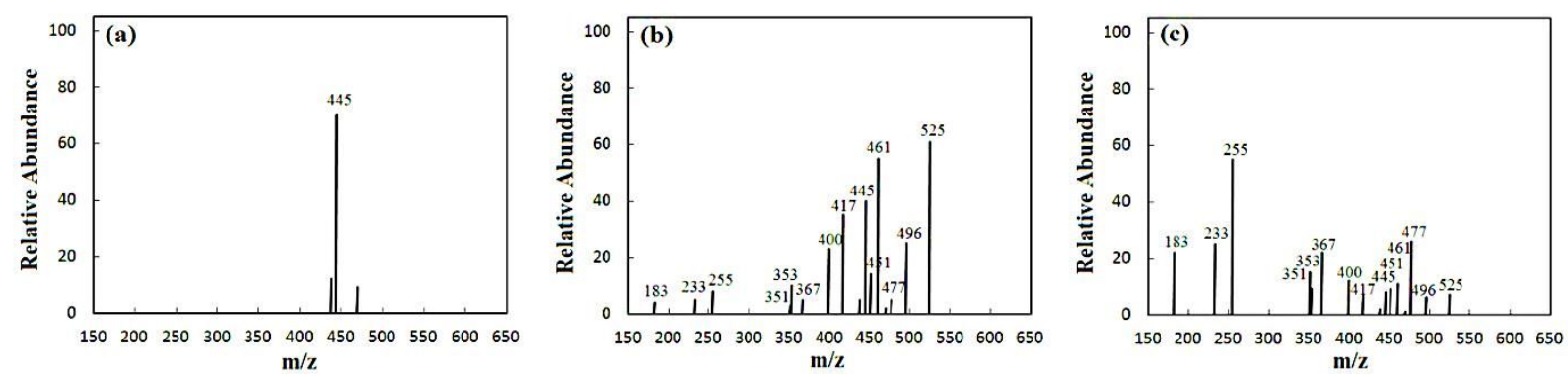

Figure S14. Mass spectra of water solutions during the sonophotocatalytic degradation of tetracycline over NU@ZIS20 nanocomposite at different reaction time: (a) 0 min, (b) 10 min, and (c) $30 \mathrm{~min}$. 


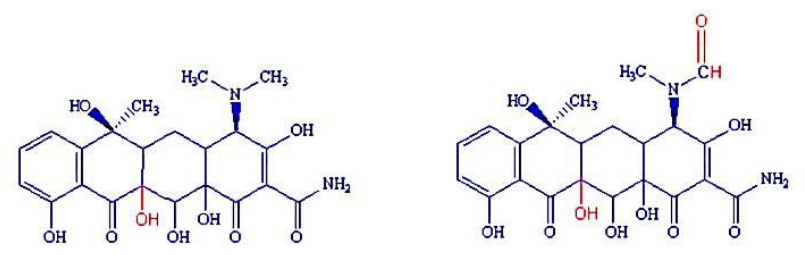

(a)
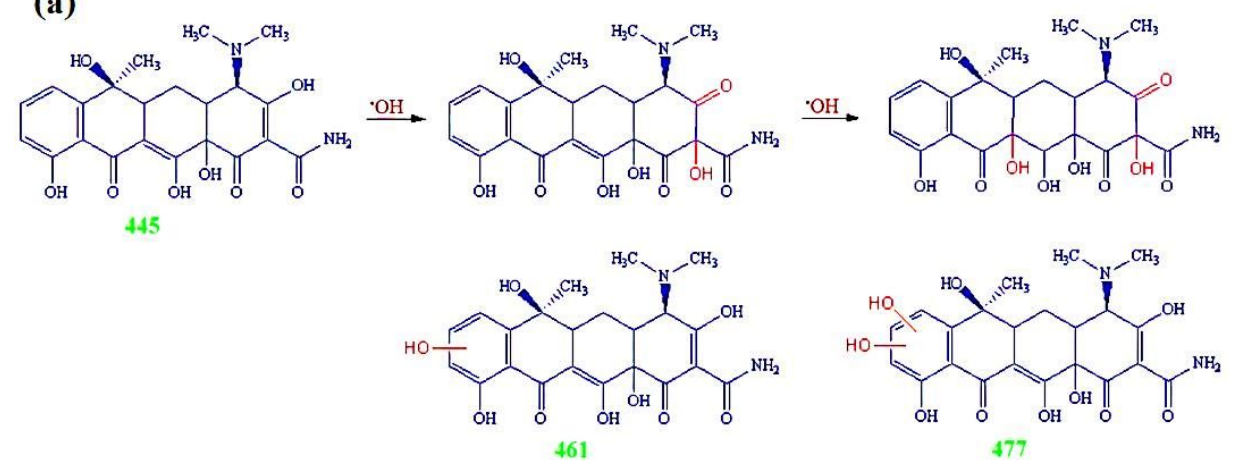

(b)

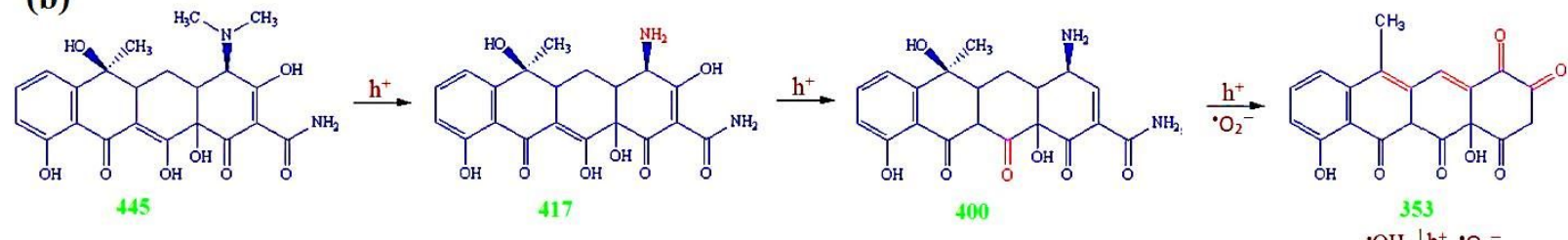

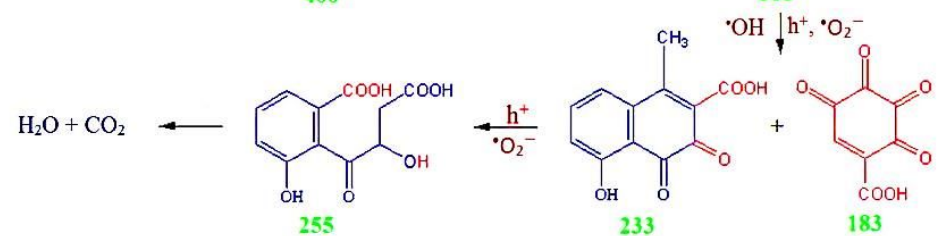

(c)

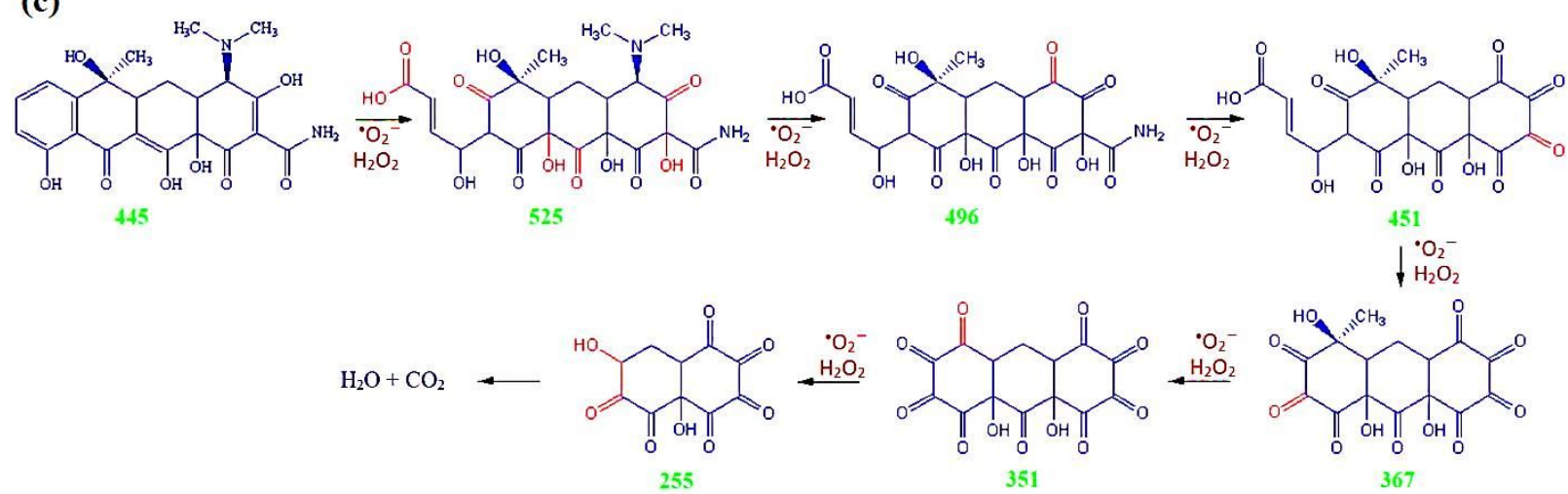

Figure S15. Possible pathways and intermediates in the sonophotocatalytic degradation of tetracycline over NU@ZIS20, established on the basis of HPLC-MS and literature data. 
Table S1. BET surface area, pore volume and pore size of samples.

\begin{tabular}{|c|c|c|c|}
\hline Sample & BET Surface area $\left(\mathrm{m}^{2} \mathrm{~g}^{-1}\right)$ & pore volume $\left(\mathrm{cm}^{3} / \mathrm{g}\right)$ & pore size $(\AA)$ \\
\hline NU-1000 & 2137 & 1.38 & 31.0 \\
\hline ZIS & 34 & 0.15 & 4.6 \\
\hline NU@ZIS20 & 1856 & 1.20 & 29.5 \\
\hline
\end{tabular}


Table S2. Comparison of photocatalytic degradation of tetracycline based on MOFs.

\begin{tabular}{|c|c|c|c|c|c|c|}
\hline Catalyst & Antibiotic & Light & $\begin{array}{l}\text { Time } \\
(\min )\end{array}$ & $\begin{array}{c}\text { Pollutant } \\
\text { concentration }\end{array}$ & $\begin{array}{c}\text { Removal } \\
\text { rate (\%) }\end{array}$ & Ref. \\
\hline NZVI/MIL-101(Cr) & Tetracycline & Visible & $340 \min$ & $200 \mathrm{mg} / \mathrm{L}$ & 90 & (1) \\
\hline Co-doped UiO-66 & Tetracycline & Sunlight & $180 \min$ & $20 \mathrm{mg} / \mathrm{L}$ & 94 & $(2)$ \\
\hline MWCNT/MIL-53 & Tetracycline & Adsorption & $160 \mathrm{~min}$ & $200 \mathrm{mg} / \mathrm{L}$ & 90 & (3) \\
\hline CuCo/MIL-101 & Tetracycline & Adsorption & $12 \mathrm{~h}$ & $100 \mathrm{mg} / \mathrm{L}$ & 83 & (4) \\
\hline $\mathrm{CdS} / \mathrm{NC}-\mathrm{T}$ & Tetracycline & Visible & $60 \mathrm{~min}$ & $40 \mathrm{mg} / \mathrm{L}$ & 83 & (5) \\
\hline ZIF-8/PAN & Tetracycline & Visible & $10 \mathrm{~h}$ & $50 \mathrm{mg} / \mathrm{L}$ & 97 & $(6)$ \\
\hline NU@ZIS20 & Tetracycline & Visible & $35 \mathrm{~min}$ & $20 \mathrm{mg} / \mathrm{L}$ & 99 & This work \\
\hline
\end{tabular}

\section{References}

(1) Hou, X.; Shi, J.; Wang, N.; Wen, Z.; Sun, M.; Qu, J.; Hu, Q. Removal of Antibiotic Tetracycline by Metal-Organic Framework MIL-101(Cr) Loaded Nano Zero-Valent Iron. J. Mol. Liq. 2020, 313, 113512.

(2) Cao, J.; Yang, Z.-H.; Xiong, W.-P.; Zhou, Y.-Y.; Peng, Y.-R.; Li, X.; Zhou, C.-Y.; Xu, R.; Zhang, Y.R. One-Step Synthesis of co-Doped UiO-66 Nanoparticle with Enhanced Removal Efficiency of Tetracycline: Simultaneous Adsorption and Photocatalysis. Chem. Eng. J. 2018, 353, 126.

(3) Xiong, W.; Zeng, G.; Yang, Z.; Zhou, Y.; Zhang, C.; Cheng, M.; Liu, Y.; Hu, L.; Wan, J.; Zhou, C.; Xu, R.; Li, X. Adsorption of Tetracycline Antibiotics from Aqueous Solutions on Nanocomposite Multi-walled Carbon Nanotube Functionalized MIL-53(Fe) as New Adsorbent. Sci. Total Environ. 2018, 627, 235.

(4) Jin, J.; Yang, Z.; Xiong, W.; Zhou, Y.; Xu, R.; Zhang, Y.; Cao, J.; Li, X.; Zhou, C. Cu and co Nanoparticles co-Doped MIL-101 as a Novel Adsorbent for Efficient Removal of Tetracycline from Aqueous Solutions. Sci. Total Environ. 2019, 650, 408.

(5) Cao, H.-L.; Cai, F.-Y.; Yu, K.; Zhang, Y.-Q.; Lü, J.; Cao, R. Photocatalytic Degradation of Tetracycline Antibiotics over CdS/Nitrogen-Doped-Carbon Composites Derived from in Situ Carbonization of Metal-Organic Frameworks. ACS Sustainable Chem. Eng. 2019, 7, 1084710854.

(6) Zhao, R.; Shi, X.; Ma, T.; Rong, H.; Wang, Z.; Cui, F.; Zhu, G.; Wang, C. Constructing Mesoporous Adsorption Channels and MOF-Polymer Interfaces in Electrospun Composite Fibers for Effective Removal of Emerging Organic Contaminants. ACS Appl. Mater. Interfaces 2021, 13, 755-764. 